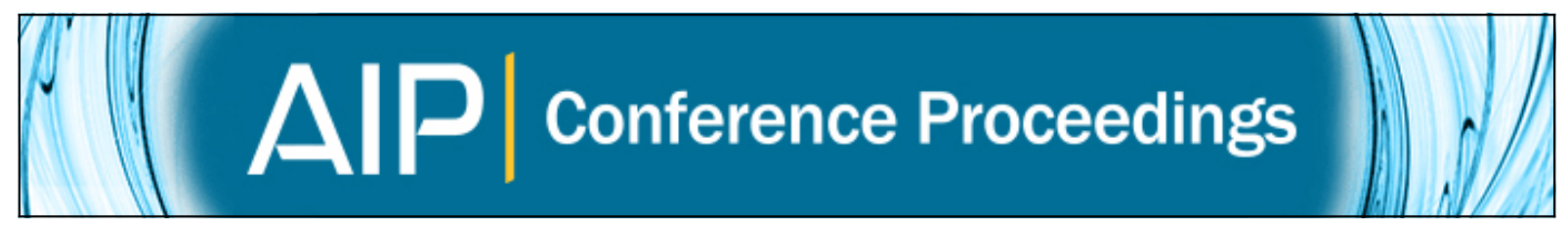

\title{
The CMS ECAL Laser Monitoring System
}

Adolf Bornheim and CMS ECAL Collaboration

Citation: AIP Conference Proceedings 867, 408 (2006); doi: 10.1063/1.2396979

View online: http://dx.doi.org/10.1063/1.2396979

View Table of Contents: http://scitation.aip.org/content/aip/proceeding/aipcp/867?ver=pdfcov

Published by the AIP Publishing

\section{Articles you may be interested in}

CMS ECAL Calibration Strategy

AIP Conf. Proc. 867, 400 (2006); 10.1063/1.2396978

Test beam results on the performance of the CMS electromagnetic calorimeter

AIP Conf. Proc. 867, 350 (2006); 10.1063/1.2396972

Crystal Properties in the Electromagnetic Calorimeter of CMS

AIP Conf. Proc. 867, 245 (2006); 10.1063/1.2396960

Observation of the trajectories of particles in process equipment by an in situ monitoring system using a laser light scattering method

J. Vac. Sci. Technol. B 16, 3339 (1998); 10.1116/1.590378

Real-time monitoring of scattered laser light by a single particle of several tens of nanometers in the etching chamber in relation to its status with the equipment

J. Vac. Sci. Technol. A 16, 1189 (1998); 10.1116/1.581257 


\title{
The CMS ECAL Laser Monitoring System
}

\author{
Adolf Bornheim, for the CMS ECAL Collaboration \\ California Institute of Technology, 1200 East California Blvd. , Pasadena, CA 91125, USA
}

\begin{abstract}
The CMS detector at LHC will be equipped with a high precision lead tungstate electromagnetic calorimeter. To ensure the stability of the calorimetric response at a level of a few per mille, every channel of the detector is monitored with a laser system. This system allows to correct for fluctuations in the detector response with high precision, in particular the expected radiation induced changes of the crystal transparency, which are on the level of several per cent at nominal luminosity. Here we report results from long term tests of the system on fully equipped supermodules of the CMS ECAL which are performed after the final step of the construction but before insertion of the modules in CMS. The in-situ monitoring strategy for the CMS ECAL is discussed in detail and the performance of the full monitoring procedure as achieved in the test beam is illustrated.
\end{abstract}

Keywords: Lead Tungstate Crystal Calorimeter, CMS ECAL, Monitoring System PACS: 29.40.Vj, 29.40.Mc,

\section{INTRODUCTION}

One of the main physics goals of the CMS detector at LHC is the search for the Higgs boson. In the mass range around $125 \mathrm{GeV}$, the decay $\mathrm{H} \rightarrow \gamma \gamma$ is of particular interest because of its clean final state. This mode however requires a very good energy resolution for the electromagnetic calorimeter to take advantage of the expected narrow decay width of the Higgs boson in this mass range. The design resolution for this calorimeter foresees a constant term in the resolution formula of $0.55 \%$. To fulfill this requirement, the CMS experiment features a high resolution crystal calorimeter utilizing lead tungstate (PWO) crystals [1]. These crystals do experience a very slight, dose rate dependent decrease of their transparency under irradiation, which recovers to a large extend in irradiation free periods. In CMS, the variation of the crystal transparency under irradiation and the corresponding decrease in light out put of the crystals will be monitored with a high precision light monitoring system. In this paper we describe the experience with the final design monitoring system in the test beam at CERN and discuss the strategy for correcting the variation in the crystal response during LHC operation.

CP867, XII International Conference on Calorimetry in High Energy Physics, edited by S. R. Magill and R. Yoshida

(C) 2006 American Institute of Physics 978-0-7354-0364-2/06/\$23.00 


\section{TRANSPARENCY CHANGE OF LEAD TUNGSTATE CRYSTALS UNDER IRRADIATION}

The mass produced lead tungstate crystals used for the CMS ECAL are radiation hard to high integrated dosage, but they experience a dose rate dependent transparency loss, as shown in Figure 1 (left). Although there is no damage to the scintillation mechanism, color centers are created during irradiation. These radiation induced color centers may annihilate at room temperature. During irradiations both annihilation and creation processes coexist and the color center density reaches an equilibrium at a level depending on the dose rate applied.
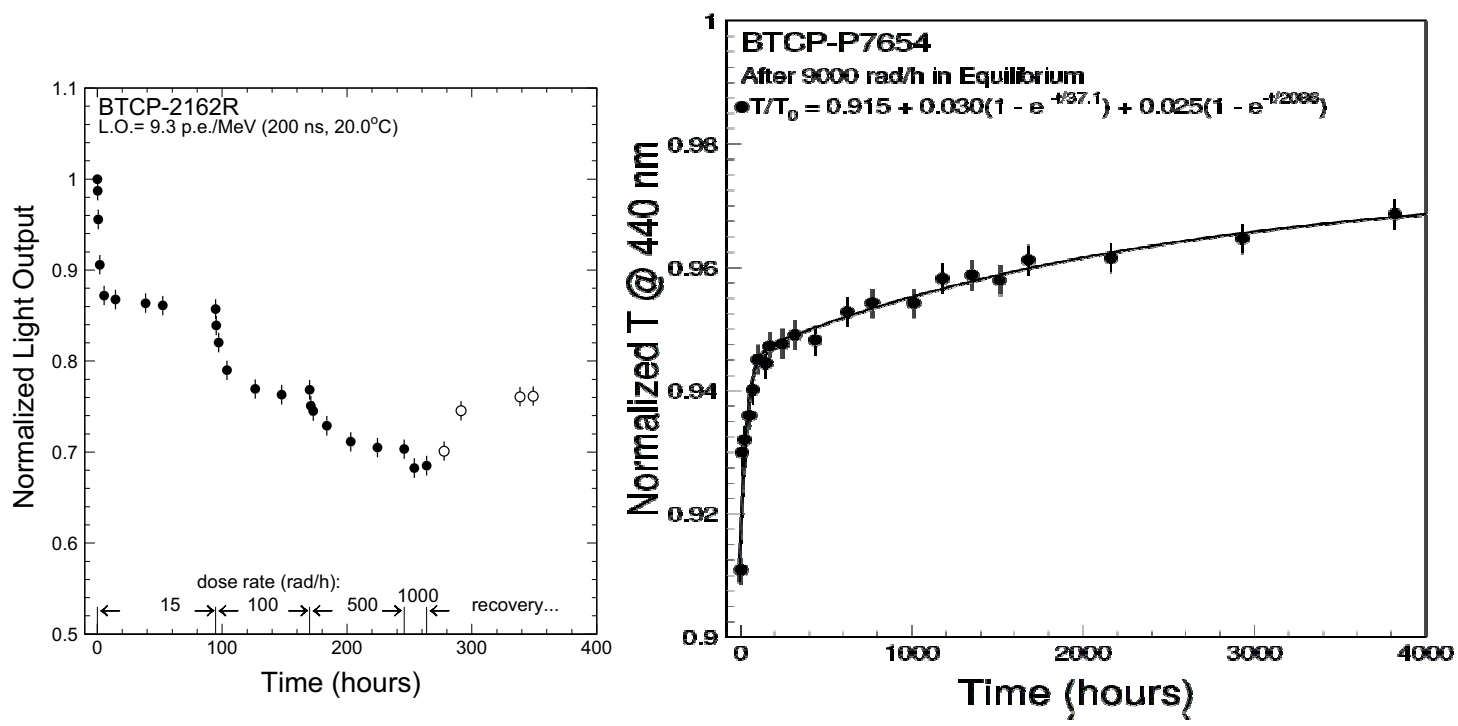

FIGURE 1. The left plot shows the change in the crystal transparency at different dose rates. Note that the transparency change reaches an equilibrium at each rate under continued irradiation. The dose rate during the first 100 hours of 15 rad per hours corresponds to the expected dose rate at LHC during low luminosity running. Note also that the relative change in the transparency is largest at small dose rates and becomes increasingly smaller at larger doserates. The right plot shows the recovery of the transparency loss after an irradiation. The recovery can be described by two time constants.

When the irradiation stops, the color center density decreases until it reaches an equilibrium again and thus the transparency change recovers. The time constants for the damage and recovery process are a crystal property and can vary to some extend. The recovery can be described by two time constants. An initial recovery phase takes place on a time scale of approximately 10 hours, followed by a slower recovery on the time scale of 1000 hours, as illustrated in Figure 1 (right). This has to be compared of the typical time constants of the LHC accelerator cycle. The luminosity operation will last for about 12 hours, followed by a refill of the machine which takes of the order one hour. The interplay between the recover time constants and the LHC operational cycle will lead to a distinct pattern in the transparency change, as illustrated in Figure 2. Since the crystals will not fully recover during the refill period of the accelerator the maximal transparency change will be limited. This will reduce the systematic effects 
related to the transparency correction since it will reduce the size of the correction on average.
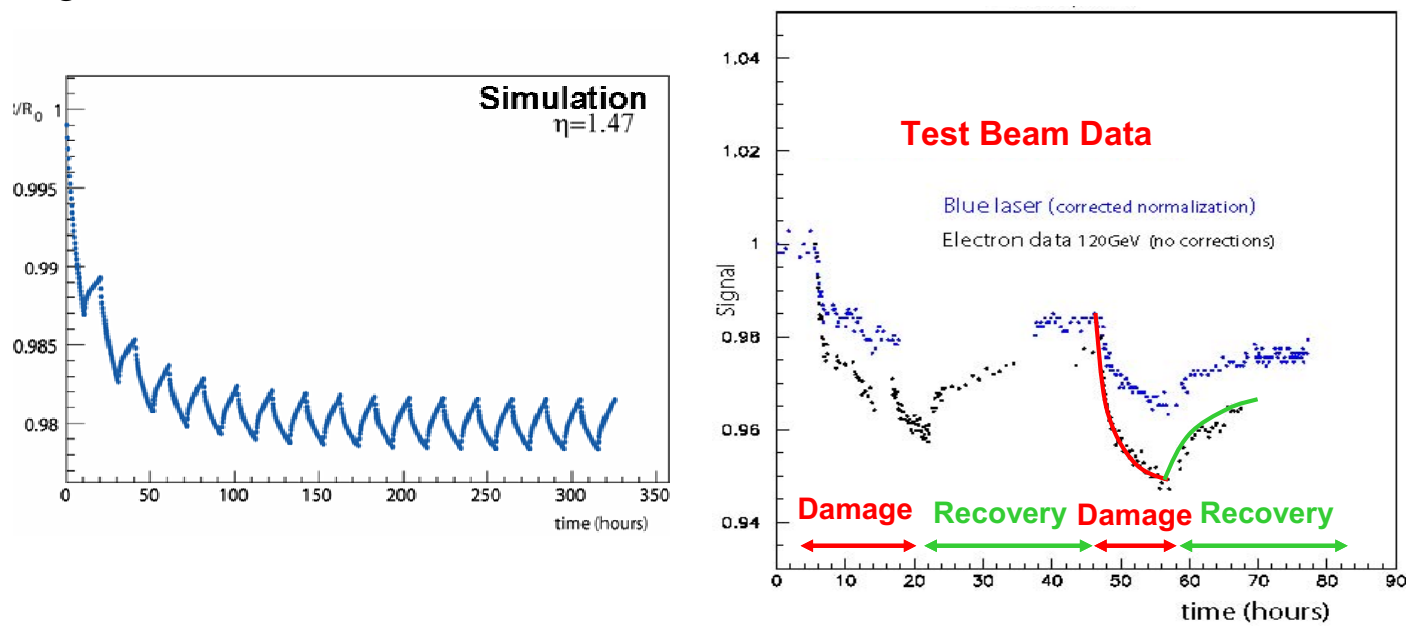

FIGURE 2. The diagram on the left from an simulation illustrates the interplay between the two time constants of the crystal recovery and the operational cycle of the LHC accelerator. Since the recovery time is much longer than one LHC cycle, the transparency change will not fully recover during refilling of the LHC. This will limit the overall fluctuations of the transparency change. The diagram on the right shows two such irradiation cycles mimicked at the test beam by repeatedly irradiating a final design CMS ECAL super module. The expected behavior can clearly be seen.

\section{THE CMS ECAL MONITORING SYSTEM}

The laser monitoring system must track the change in the transparency precisely enough to maintain the constant term in the ECAL resolution of $0.55 \%$. This requires a measurement of the transparency with an accuracy of better than $0.2 \%$. The transparency in each crystal will have to be measured approximately every 30 minutes during LHC operation to account for the fast initial change at the beginning of an irradiation cycle.
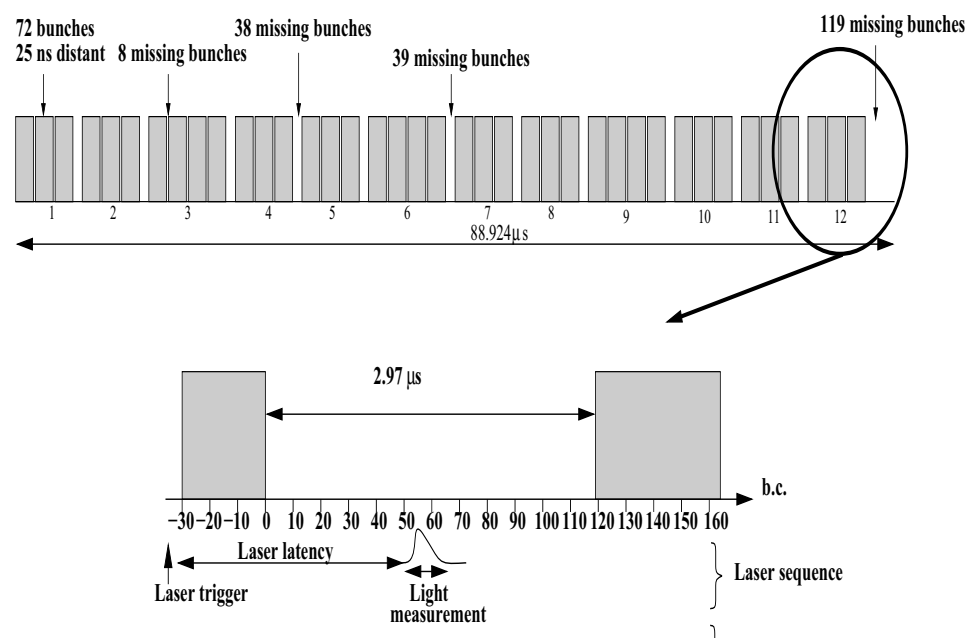

FIGURE 3. The diagram illustrates the continuous laser monitoring during LHC operation. Taking advantage of a $3 \mu$ s gap in the LHC bunch train laser pulses are injected at a rate of $100 \mathrm{~Hz}$. 
To achieve this, the monitoring has to be performed while the data taking of the detector is ongoing. This can be realized by taking advantage of gaps in the LHC bunch train which occur once every $90 \mu \mathrm{s}$. In Figure 3 the time structure of the LHC bunch train is illustrated. The laser system will be synchronized with the LHC clock cycle via the CMS trigger system and send laser pulses during the extended gaps. To allow this operational mode the laser source has to fulfill a number of design parameters. In particular the laser pulse width must be shorter than $40 \mathrm{~ns}$ and stable to better than $10 \%$ to match the shaping of the ECAL electronics. Furthermore must the pulse timing be stable to a few ns to allow a synchronization which the LHC clock and the detector readout. Finally, the pulse energy must be on the order of $1 \mathrm{~mJ}$ to provide sufficient light to large groups of crystals to ensure that the entire ECAL can be monitored in 30 minutes. The pulse energy also has to be stable to better than $10 \%$ to prevent possible non-linear effects in the reference system against which the signals of the individual crystals are compared. To achieve all these performance requirements a dual-stage laser system is used $[3,4]$. Two such laser systems provide two different wavelengths, on at the peak of the scintillation light wavelength at $440 \mathrm{~nm}$, one far away in the red wavelength range to allow for systematic cross checks. These two systems plus an spare backup system for the blue laser light source distribute the light pulses via a system of optical switches to the ECAL super modules, as shown in Figure 4.

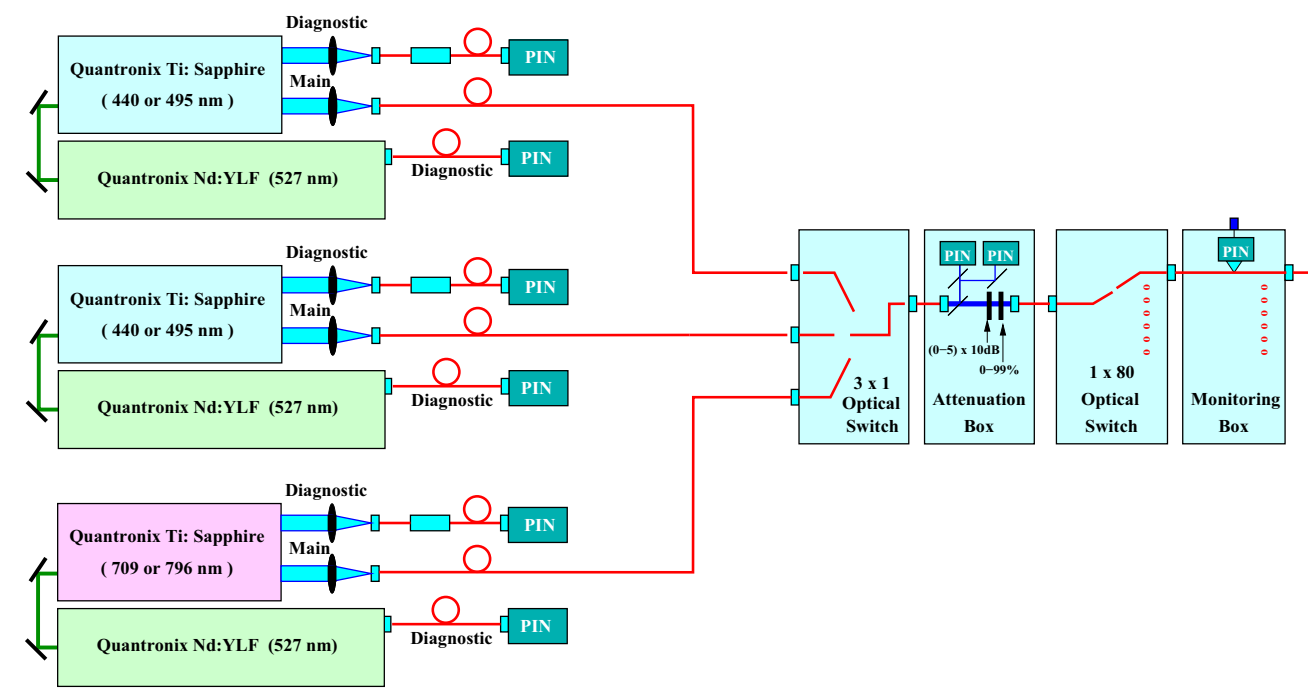

FIGURE 4. The diagram shows the layout of the CMS ECAL laser monitoring source. Two laser systems provide light at $440 \mathrm{~nm}$ and $800 \mathrm{~nm}$ as primary and secondary monitoring wavelengths. The $440 \mathrm{~nm}$ system has an identical spare system as a backup. A system of optical switches allows to distribute the light to one half of a super module at a time. All half super modules of the entire ECAL will be scanned once within 30 minutes.

Inside the super module the laser light is distributed via optical fibers and a fanout system to each individual crystal. The optical fibers enter the crystals from the front in the barrel part of ECAL and from the back in the endcaps. In parallel light is sent to 
reference photo diodes inside the supermodule. The relative difference seen in the photodetectors of the ECAL with repect to the reference diodes measures the relative transparency change in the crystals. More details of the on-detector part of the monitoring system can be found in reference [5].

\section{TRANSPARENCY CORRECTION WITH THE CMS ECAL LASER MONITORING SYSTEM}

The feasibility of the radiation damage monitoring with the desired precision has been demonstrated in the test beam [6]. One important measure of the performance is the stability of the monitoring system response over time in the absence of any radiation. This is a measure of the resolution of the system which sets the scale for the minimal transparency change which can be detected. The design goal for the CMS ECAL monitoring system is to be able to track transparency changes on the level of better than $0.2 \%$. The second benchmark is the ability of the system to correct back transparency changes induced by irradiation. This is verified in the test beam by irradiating crystals in a high energy electron beam with dose rates similar to the ones expected in CMS. During the irradiation the crystals are calibrated with the electron beam in regular time intervals, followed by laser monitoring runs during which the transparency is measured. The response to the electrons is then corrected using the transparency measurements of the laser system. Aside from the stability of the monitoring system there are several other issues which impact the performance of this correction procedure. The most important one is the relation between the relative change of the laser monitoring signal and the relative change of the response to electrons. This relation can be described by a linear function for small transparency changes, as shown in the left plot of Figure 4.
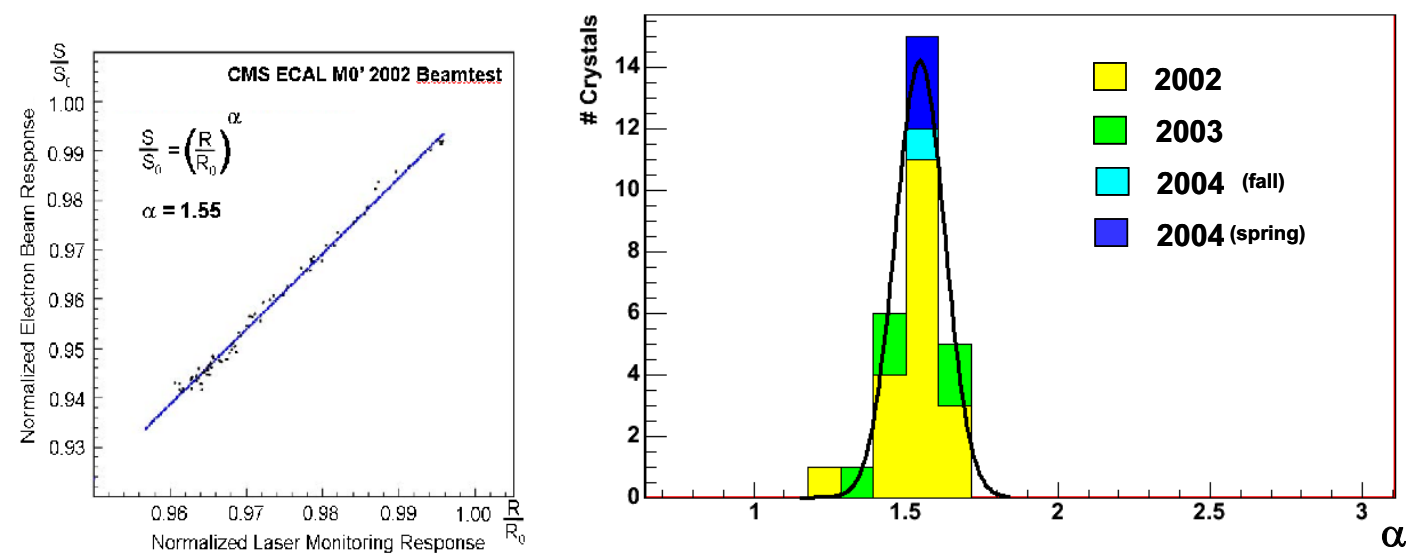

FIGURE 5. The left plot shows the linear correlation between the relative loss of the laser monitoring response and the relative loss of the response to electrons. The slope $\alpha$ of this linear correlation is quite uniform for crystals produced under the same conditions. The plot on the right shows the slope from 28 crystals produced by BTCP, the main supplier of crystals for the CMS ECAL. The distribution has an RMS of about $5 \%$. 
Thus, only a scaling factor is needed to determine the correction factor from the relative change as measured by the laser system. This scaling factor $\alpha$ is found to be the same within about $5 \%$ for crystals produced under the same conditions, as shown in the right plot of Figure 4. For a total of 28 crystals the parameter $\alpha$ has been measured in various test beam campaigns and found to be the same in all crystals within $5 \%$. There are indications that the orientation of the crystal optical axis with respect to the crystal geometrical shape do impact in the scaling factor $\alpha$. At CMS the transparency change in the LHC low luminosity running will be on the level of a few percent. At this level it is sufficient to know the average value of the parameter $\alpha$ as determined in the test beam. Procedures to extract the parameter $\alpha$ in situ using physics events are under consideration.

\section{PERFORMANCE OF THE MONITORING SYSTEM}

In Figure 5 we show an example of an irradiation cycle in the test beam. A final design super module was irradiated with $120 \mathrm{GeV}$ electrons at does rates equivalent to four times the expected does rate at LHC in low luminosity operation. During the irradiation the crystals exposed to the beam were calibrated repeatedly with the electron beam. In parallel the transparency was monitored with the laser monitoring system. Over a period of six hours we observe a decrease of the raw response to electrons of about $2 \%$.

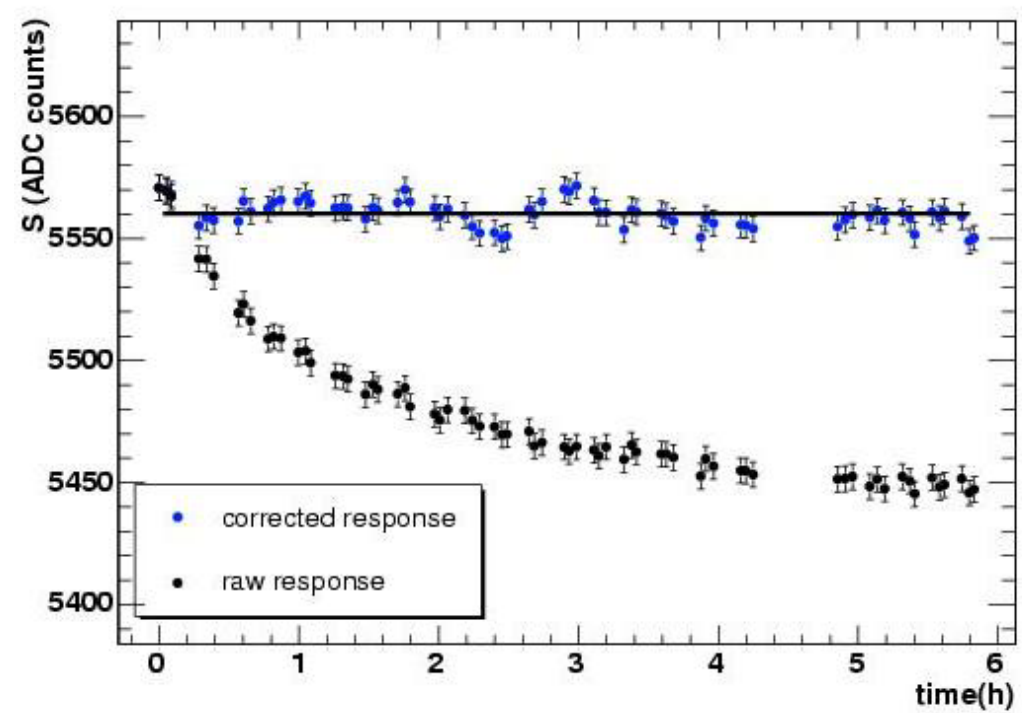

FIGURE 6. The raw ECAL response to $120 \mathrm{GeVelectrons}$ during an irradiation period on a super module in the test beam. The response is the sum of nine crystals centered around the crystal hit by the beam. The beam rate was set such that the radiation level corresponds to four times the expected radiation level at the LHC. The crystal response drops by about 2 percent and then approaches a plateau. The data has then be corrected using the laser monitoring data. The individual calibration points are within $0.15 \%$ of the initial calibration. 
After applying a correction based on the measurements from the laser monitoring system the individual calibration points are within $0.15 \%$ of the initial calibration before irradiation [7]. This value is achieved for a set of four crystals all irradiated in the same was as shown in the example in Figure 5. For this correction the average value for the parameter $\alpha$ as determined from Figure 4 was used. This is to be compared to the design value for the constant term of the energy resolution of the CMS ECAL which is $0.55 \%$. The effect of the transparency change correction is well below other effects contributing to the overall constant term, in particular the intercalibration to be performed in situ [8].

\section{SUMMARY}

A final design laser monitoring system of the CMS ECAL has been tested in the test beam. With this monitoring system the radiation induced transparency change in the crystals of the CMS ECAL can be measured and corrected for. The residual effect after the correction of the transparency change is found to be less than $0.15 \%$. This is well within design specifications and negligible compared to other contributions to the constant term of the resolution of $0.55 \%$.

\section{ACKNOWLEDGMENTS}

I would like to thank the entire ECAL collaboration for their outstanding effort, R.-Y. Zhu and the CALTECH team for their superb work on the laser system and the organizers of the XII International Conference on Calorimetry in High Energy Physics at Chicago.

\section{REFERENCES}

1. "The Electromagnetic Calorimeter Technical Design Report", CMS Collaboration, CERN/LHCC 97-33, 1997

2. "Radiation Induced Color Centers and Light Monitoring for Lead Tungstate Crystals", X. Qu, L. Zhang, R.-Y, Zhu, IEEE Trans. Nucl. Sci. NS-47 (2000).

3. "Performance of the Monitoring Light Source for the CMS Lead Tungstate Crystal Calorimeter", IEEE Trans. Nucl. Sci.Vol. 52 No. 4 (2005) 1123--1130.

4. "ECAL Monitoring Light Source at H4", D. Bailleux, A. Bornheim, L. Y. Zhang, K. J. Zhu, R.-Y. Zhu, and D. Liu, CMS IN 2003/045.

5. "Laser monitoring system for the CMS lead tungstate crystal calorimeter", M. Anfreville et. al., to appear in Nucl. Instr. And Meth.

6. "The CMS ECal laser monitoring system.", A. Bornheim, in proceedings of the 9th ICATPP Conference on Astroparticle, Particle, Space Physics, Detectors and Medical Physics Applications, Como, Italy.

7. "Analysis of the response evolution of the CMS electromegnetic calorimeter under electron and pion irradiation", A. Ghezzi et. al., CMS NOTE-2006/038

8. “CMS ECAL Calibration Strategy”, G. Daskalakis, these Proceedings 